Research Paper

\title{
Non-invasive Brain Stimulation and Prism Adaptation in Art Constructive Errors in Painting
}

\author{
Shole Vatanparasti $^{1 *}$ (D), Anoshirvan Kazemnejad² ${ }^{2}$, Shahram Oveisgharan ${ }^{3}$ (i) \\ 1. Department of Rehabilitation, Institute for Cognitive Science Studies, Tehran, Iran. \\ 2. Department of Biostatistics, Faculty of Medical Sciences, Tarbiat Modares University, Tehran, Iran \\ 3. Department of Neurology, Rush Alzheimer's Disease Center, Rush University Medical Center, Chicago, United States.
}

\begin{tabular}{|c|c|}
\hline $\begin{array}{l}\text { Use your device to scan } \\
\text { and read the article online }\end{array}$ & ditation Vatanparasti, S., Kazemnejad, A., \& Oveisgharan, S. (2023). Non-invasive Brain Stimulation and Prism Ad- \\
\hline 口ipirita & $\begin{array}{l}\text { aptation in Art Constructive Errors in Painting. Basic and Clinical Neuroscience, 14(1), 143-154. http://dx.doi.org/10.32598/ } \\
\text { bcn.2021.2207.1 }\end{array}$ \\
\hline 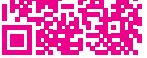 & 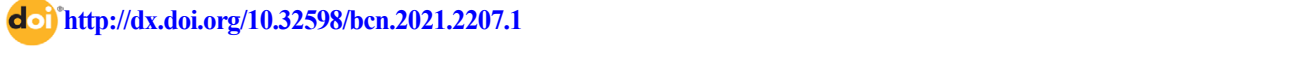 \\
\hline
\end{tabular}

Article info:

Received: 13 Nov 2019

First Revision: 19 Oct 2020

Accepted: 15 Jun 2021

Available Online: 01 Jan 2023

Keywords:

Rehabilitation, Neglect, Painting, Coloring, Prism, Brain stimulation

\begin{abstract}
A B S T RA C T
Introduction: This study aimed to investigate the influence of neglect and the effect of prism adaptation (PA) combined with continuous Theta-burst transcranial magnetic stimulation (cTBS) on the art constructive errors in painting rehabilitation of stroke patients with neglect.

Methods: Fourteen patients with neglect and art constructive errors in painting secondary to stroke were randomly assigned to the rehabilitation group and received PA combined with the inhibitory protocol of cTBS over the intact parietal cortex; the control group received PA combined with sham cTBS for two weeks in ten daily sessions. Patients were assessed for art constructive errors in painting in figure copying test (FCT), and coloring test (CT) before and after the intervention. Art constructive errors in painting were classified into omission, deformation, size, neglect of warm colors, and perseveration of errors. Neglect was evaluated using the line bisection task (LBT), figure copying test (FCT), and coloring test (CT).
\end{abstract}

Results: All patients showed a significant improvement in art constructive errors in painting (measured using the pattern of painting' errors in FCT and CT), and neglect (measured using LBT, FCT, and CT $(\mathrm{P}<0.05)$. Omission, neglect of warm colors, and deformation were the most frequent errors.

Conclusion: Neglect and rehabilitation influence the painting system in stroke patients. Both approaches improved art constructive errors in painting and neglect symptoms. 


\section{Highlights}

- Both therapeutic approaches (cTBS+PA and PA) showed significant implicit recovery in art constructive errors in painting and neglect without practice.

- Visuospatial unilateral spatial neglect appears to specifically affect the art system of patients with stroke.

- Art constructive errors in painting patterns were omission, deformation, size discrepancy, neglect of warm colors, and perseveration.

\section{Plain Language Summary}

Art is a human communication tool. However, there is limited research on therapy for stroke adult patients with acquired art constructive errors in painting. Nevertheless, no study to date has examined the relation between art-specific constructive errors in painting and spatial neglect and rehabilitation without painting practice. The present study detected the pattern of art constructive errors in painting before intervention and investigated rehabilitation with prism adaption alone and prism adaption combined with continuous Theta-Burst Transcranial Magnetic Stimulation to treat art constructive errors in painting in patients with neglect.

\section{Introduction}

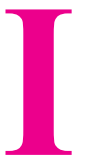

njury to the right hemisphere of the brain often associated with the neurological disability of function and neglect-induced cognitive impairment in drawing, coloring, and copying, is known as art constructive errors in painting (Rhee, et al, 2016; Rode, et al., 2017). Art constructive errors are among the spatial disorders of painting and commonly include missing elements, omission errors, change in size errors, visuospatial destruction mistakes, and neglect of warm colors faults. These errors have been observed in patients, such as those with professional artists, with damage to the right side of the brain (Annoni, et al., 2005; Bäzner \& Hennerici, 2007). Particularly, right-brain stroke-related disturbance in painting demonstrates various forms associated with the distinctive painting system of a given art. For example, some professional painters had difficulty in painting in terms of deformation figures, disruption in landscapes, and disruption in the estimate of depth and distance (Annoni, et al., 2005; Bäzner \& Hennerici, 2007; Chen et al., 2016; Mazzucchi et al., 2013; Pasqualini \& Pasqualini, 2012). However, these art constructive errors in painting are not quite understood. The errors may be affected by visuospatial processing, have a convened array of art constructive errors in painting, and require a particular rehabilitation strategy of their art constructive errors systems (Bäzner \& Hennerici, 2007; Vaes et al., 2018; Vallar et al., 2006). Currently, clinicians have several options to consider when choosing interventions for those living with a deficit in visuospa- tial processing and unilateral neglect, including mirror therapy, prism adaption (PA), and non-invasive brain stimulation (Azouvi, et al., 2017). Prism intervention is a visuospatial processing rehabilitation that has been shown to have a promising therapeutic effect on multiple aspects of visuospatial neglect, motor-related symptoms of spatial neglect, as well as the performance of activities (Hreha et al., 2018). Non-invasive brain stimulation, transcranial magnetic stimulation (TMS) is another approach treatment that has been effective in visuospatial unilateral neglect recovery. At least one study showed higher efficacy of continuous theta-burst transcranial magnetic stimulation (cTBS) compared to other patterns of TMS (Cazzoli et al., 2012; Cotoi, et al., 2019; Yang et al., 2015). Therefore, there is a need for more effective approaches for stroke rehabilitation; for example, a combination of PA combined with cTBS. However, the effect combined PA and cTBS has not been studied on changes in specific art constructive errors in painting and visuospatial unilateral neglect.

Accordingly, patterns in art constructive errors in painting were detected to improve patterns in the error in painting. In this study, we examined the relationship between art constructive errors in painting and neglect in stroke patients before and after rehabilitation. We hypothesized that cTBS can increase prism adaption effects on improving art constructive errors in painting and visuospatial unilateral neglect test scores. 


\section{Materials and Methods}

This pilot study was conducted in Shariati Hospital, Tehran University of Medical Sciences, Iran, from August 2017 to November 2018.

\section{Subjects}

Fourteen individuals enrolled for rehabilitation in neglect and art constructive errors in painting. Stroke patients with neglect and art constructive errors in painting, verified by MRI, clinical examination, and paper-pencil evaluation (line bisection task (LBT), figure copying test (FCT), and coloring test (CT)), were enrolled and provided informed consent for participation. A total of 14 patients with neglect and art constructive errors in painting secondary to stroke were randomized via block randomization method to two groups for rehabilitation. They were tested for art constructive errors in painting using FCT, and CT. They were also asked to attend ten sessions over a 2-week period of rehabilitation with prism adaption (Ten Brink et al., 2015) combined with non-invasive brain stimulation. The prism glasses with ten degrees of visual field displacement with adaptation by mirror training were given to the participant. The stroke patient was sitting near a table, on which a vertical mirror box $(35 \times 35 \times 35 \mathrm{~cm})$ was placed. They observed the reflection of the right intact hand as the movement of the left hand in the mirror for 20 minutes ( $\mathrm{Ng}$ et al., 2015). In addition to prism adaption, one group of stroke patients received continuous theta burst stimulation over the left parietal (P3) cortex for two weeks in ten sessions, and the other group received sham continuous theta burst stimulation over the same (P3) cortex for two weeks in ten sessions. The measurements were done before and after rehabilitation. No follow-up was performed. Neglect patients with art constructive errors in painting were unaware of the group assignments; they were informed that they are going to undergo treatment for their art constructive errors in painting and visuospatial unilateral neglect. However, the cognitional therapist was aware of the neglect of patients' group allocation. The inclusion criteria consisted of neglect due to stroke, art constructive errors in painting, having suffered a rightbrain stroke, and being right-handed. The exclusion criteria were age less than 18 and more than 80 years, brain trauma, implanted heart pacemaker, a previous history of copying and coloring or painting deficit, epilepsy, cerebral edema, and intense pain. All 14 participants were right-handed; $10(70 \%)$ were men and 4(30\%) were women aged between 46 and 79 years. In terms of the type of stroke, $6(40 \%)$ participants had ischemic cerebral infarction and the stroke onset date was before six months prior to the randomization in six $(40 \%)$ patients. There was no difference in demographic baseline characteristics in terms of age, sex, education, acute stage (stroke onset date was before six months), chronic stage (stroke onset date was after six months), type of stroke, and outcomes before the intervention (Table 1).

Assessment of art constructive errors in painting, and neglect and measurement technique

All participants were evaluated for art constructive errors in painting and neglect before and after treatment. Art constructive errors in painting were measured to detect, size error, perseveration, deformation, and neglect in warm colors using the FCT (Johannsen \& Karnath, 2004) and CT (Blanke \& Pasqualini, 2012). Unilateral neglect was measured using the LBT (Bonato et al., 2008; Guariglia et al., 2014).

They were also requested to attend for two weeks in ten daily sessions of the prism adaption (PA) combined with continuous theta-burst transcranial magnetic stimulation (cTBS) in the experimental group and PA combined with sham cTBS in the control group.

In the art constructive errors of the painting task, all participants completed the FCT and CT on an A4-sized plain paper before and after the rehabilitation. No time limit was considered for the test. Only the art constructive (visuospatial) errors were evaluated as coloring and copying of painting, and semantic art of painting errors or professional painting in the art errors were not assessed. Each error was scored as one.

Previous studies have shown the classification of art constructive errors in painting into deformation, omission, and neglect of warm colors (using cold colors) in accordance with the criteria determined by Olaf Blanke et al. (Blanke \& Pasqualini, 2012). We used new suggestions for the detection of the classification of art constructive errors in the painting system. Constructive painting errors were further classified into omission, deformation, small size error, neglect of warm colors, and perseveration errors in the FCT and CT (Figures 1 and 2). Deformation errors are the creation of a nonexistent form of copying or coloring. Omission errors mean that the painter ignores more than $50 \%$ of the space in the square of coloring and deletes one part of the landscape from the shape in FCT. Visuospatial size error means the painter changes normal size painting to small size painting of CT and FCT. Neglect of warm colors means that the painter changes colors of warm (yellow, light green, and red) to colors of cold, which is characterized by cold, 
Table 1. Patient's characheristics†

\begin{tabular}{|c|c|c|c|c|c|c|c|}
\hline $\begin{array}{l}\text { N/Interven- } \\
\text { tion }\end{array}$ & Age (y) & $\begin{array}{c}\text { Sex } \\
(M / F)\end{array}$ & $\begin{array}{l}\text { Grade } \\
\text { School } \\
\text { Education }\end{array}$ & $\begin{array}{c}\text { Region } \\
\text { of the Stroke: } \\
\text { P,T,F,O,IC, } \\
\text { TH }\end{array}$ & $\begin{array}{l}\text { Time } \\
\text { Since } \\
\text { Stroke } \\
\text { Onset }\end{array}$ & $\begin{array}{l}\text { Type of the } \\
\text { Stroke }\end{array}$ & $\begin{array}{l}\text { Art Constructive Errors in } \\
\text { Painting }\end{array}$ \\
\hline 1) $\mathrm{PA}$ & 67 & M & 16 & RT, RF, RP, TH & Chronic & Hemorrhage & $\begin{array}{l}\text { Size, deformation, neglect } \\
\text { of warm colors, omission }\end{array}$ \\
\hline 2) $\mathrm{PA}$ & 68 & $\mathrm{M}$ & 9 & $\mathrm{RF}, \mathrm{RT}$ & Chronic & Hemorrhage & $\begin{array}{c}\text { Size, deformation, neglect } \\
\text { of warm colors, omission, } \\
\text { perseveration }\end{array}$ \\
\hline 3) $\mathrm{PA}$ & 63 & $\mathrm{M}$ & 9 & $\mathrm{RO}, \mathrm{RP}$ & Acute & Ischemic & $\begin{array}{l}\text { Size, deformation, neglect } \\
\text { of warm colors, omission }\end{array}$ \\
\hline 4) $\mathrm{PA}$ & 70 & M & 16 & $\mathrm{RT}, \mathrm{RF}$ & Chronic & Ischemic & Size, deformation, omission \\
\hline 5) PA & 65 & $M$ & 12 & RT & Chronic & Ischemic & Size, deformation, omission \\
\hline 6) $\mathrm{PA}$ & 46 & $\mathrm{~F}$ & 16 & RTH & Acute & Hemorrhage & Size \\
\hline 7) $\mathrm{PA}$ & 79 & $\mathrm{~F}$ & 0 & $\mathrm{RP}$ & Acute & Ischemic & $\begin{array}{l}\text { Size, deformation, neglect } \\
\text { of warm colors, omission }\end{array}$ \\
\hline 1) $C T B S+P A$ & 53 & $\mathrm{M}$ & 11 & $\mathrm{RP}$ & Chronic & Ischemic & $\begin{array}{l}\text { Size, deformation, neglect } \\
\text { of warm colors, omission }\end{array}$ \\
\hline 2) $C T B S+P A$ & 77 & $\mathrm{M}$ & 0 & $\mathrm{RT}, \mathrm{RP}, \mathrm{RO}$ & Chronic & Ischemic & Size, deformation, omission \\
\hline 3) $C T B S+P A$ & 77 & $\mathrm{M}$ & 5 & IN & Acute & Ischemic & $\begin{array}{l}\text { Size, deformation, neglect } \\
\text { of warm colors, omission }\end{array}$ \\
\hline 4) $C T B S+P A$ & 70 & $\mathrm{M}$ & 16 & $\mathrm{RF}, \mathrm{RP}, \mathrm{RT}$ & Chronic & Hemorrhage & $\begin{array}{l}\text { Size, deformation, omis- } \\
\text { sion, perseveration }\end{array}$ \\
\hline 5) $\mathrm{CTBS}+\mathrm{PA}$ & 67 & $\mathrm{~F}$ & 0 & RT,RTH & Acute & Hemorrhage & $\begin{array}{l}\text { Size, deformation, neglect } \\
\text { of warm colors, omission }\end{array}$ \\
\hline 6) $C T B S+P A$ & 62 & $\mathrm{M}$ & 12 & $R F, R T, R P$ & Acute & Hemorrhage & $\begin{array}{l}\text { Size, deformation, omis- } \\
\text { sion, perseveration }\end{array}$ \\
\hline 7) $\mathrm{CTBS}+\mathrm{PA}$ & 67 & $\mathrm{~F}$ & 12 & $R T, R P, R F$ & Chronic & Ischemic & $\begin{array}{c}\text { Size, deformation, neglect } \\
\text { of warm colors, omission, } \\
\text { perseveration }\end{array}$ \\
\hline $\begin{array}{c}\mathrm{PA} \\
\text { Mean } \pm \mathrm{SD} \\
\text { cTBS+PA } \\
\text { Mean } \pm \text { SD) } \\
\mathrm{P}^{+}\end{array}$ & $\begin{array}{c}65.42 \pm 9.98 \\
67.57 \pm 8.44 \\
P=0.67\end{array}$ & $p=0.72$ & $\begin{array}{c}11.00 \pm 5.84 \\
8.00 \pm 6.35 \\
P=0.35\end{array}$ & $\begin{array}{c}F P=0.29 \\
O P=0.76 \\
\text { TH } P=0.50 \\
\text { T } P=0.50 \\
\text { IN } P=0.50\end{array}$ & $P=0.70$ & $P=0.70$ & $\begin{array}{c}5.28 \pm 1.60 \\
5.24 \pm 0.53 \\
P=0.83\end{array}$ \\
\hline
\end{tabular}

†Dichotomous variables are compared with Fisher's exact test and quantitative variables with t-test.

NEUR SCIENCE

Abbreviations: P: Cortex parietal; T: Cortex temporal; F: Cortex frontal; R: Right; IN: Internal capsule; TH: Thalamus; O: Occipital; CTBS: Continuous theta-burst transcranial magnetic stimulation; PA: Prism adaptation; Chronic: Time since stroke onset $>6$ months; Acute: Time since stroke onset $<6$ months.

hard, and metallic colors, such as gray, black, dark green, brown, and marine blue. Perseveration error is related to the repetition and addition to the painting (Figures 1 and 2). In the CT, the stroke patients were asked to color a multi-object scene consisting of miniature style on an A4-sized plain paper. The coloring section ratio was computed from the ratio of color omitted on the left side of A4 paper to the total score of coloring canceled for neglect evaluation. We also calculated another score, which was the ratio of art constructive errors in the painting by the patient.
In the FCT, the stroke patients were asked to copy a multi-object scene consisting of five figures on an A4 paper. The omission of at least one of the left-sided features of each figure was scored as one, the omission of each whole figure was scored as two, and one preservation point was given when left-sided figures were drawn on the right side. Each perseveration was scored as one. The maximum score was ten for neglect evaluation. We also calculated another score, which was the ratio of art constructive errors in the painting by the patient. 
Table 2. Art constructive errors in painting, and visuospatial neglect measurement before and after the rehabilitation.

\begin{tabular}{|c|c|c|c|c|c|c|}
\hline \multirow{3}{*}{ Outcome } & \multicolumn{4}{|c|}{ Mean $\pm S D$} & \multirow{2}{*}{\multicolumn{2}{|c|}{ Repeated ANOVA }} \\
\hline & \multicolumn{2}{|c|}{ Before intervention } & \multicolumn{2}{|c|}{ After intervention } & & \\
\hline & PA+cTBS & PA & PA+cTBS & PA & Time & GroupxTime Factor \\
\hline Line Bisection test & $33.70 \pm 15.65$ & $27.62 \pm 11.69$ & $6.70 \pm 11.66$ & $5.67 \pm 5.75$ & $\mathrm{P}<0.001^{*}$ & $P=0.57$ \\
\hline Coloring test & $65.42 \pm 10.27$ & $43.71 \pm 24.47$ & $1.57 \pm 4.15$ & $0.71 \pm 1.88$ & $P<0.001^{*}$ & $P=0.05$ \\
\hline Figure copying test & $6.14 \pm 1.21$ & $3.71 \pm 2.75$ & $1.28 \pm 1.79$ & $0.57 \pm 0.97$ & $P<0.001^{*}$ & $P=0.11$ \\
\hline $\begin{array}{l}\text { Art constructive errors } \\
\text { in painting }\end{array}$ & $5.42 \pm 0.53$ & $5.28 \pm 1.40$ & $1.14 \pm 1.04$ & $1.85 \pm 2.11$ & $P<0.001^{*}$ & $P=0.22$ \\
\hline
\end{tabular}

"Significant difference between pre-intervention and post-intervention at $\mathrm{P}<0.05$ by repeated-measures ANOVA (time factor). "Significant difference between groups at $\mathrm{P}<0.05$ by repeated-measures ANOVA (group $\times$ time factor)

Abbreviations: cTBS: Continuous Theta-burst transcranial magnetic stimulation; PA: Prism adaptation.

In the LBT, patients were instructed to bisect 40 horizontal lines including ten lines on the right side, 18 lines on the middle side, and 12 lines on the left side of the page. The absolute distance between the patient's bisection and the midpoint straight lines were computed.

\section{TMS intervention}

We used a MagPro X100 machine (Magventure Company, Farum, Denmark) equipped with a commercially available figure-of-eight coil for cTBS. The experimental group received continuous theta-burst Stimulation (cTBS). The cTBS inhibitory protocol was 801 pulses in three bursts at $30 \mathrm{~Hz}$ and was repeated every $100 \mathrm{~ms}$ (5 $\mathrm{Hz}, \theta$ rhythm) with $80 \%$ of RMT. The cTBS inhibitory protocol was applied in P3 on the intact parietal (P3), left side based on the EG 10/20 system in ten sessions over a 2-week period (Yang et al., 2015). The control group underwent sham magnetic stimulation by tilting the coil vertically $\left(90^{\circ}\right)$ the same as the experimental true stimulation group (Rossi et al., 2007). The participants in both groups received intervention for days ten per week for two weeks. Participants were blind to the type of therapy they received. These stroke patients tolerated cTBS treatment using 8-coil without the incidence of any complications. We used a safety guideline for the inhibitory protocol (Rossini et al., 2015).

\section{Statistical analysis}

Student's t-test and Fisher's exact test were used to comparing the groups at baseline (PA combined with cTBS vs. PA alone) for continuous and dichotomous variables, respectively. Then, repeated-measures analy- sis of variance (ANOVA) was performed between the values of LBT, FCT, CT, and total art constructive errors in painting, with the group (PA combined with cTBS vs. PA alone) as between-subject main factor and time (posttreatment vs. pre-treatment) as the within-subject main factor. In each ANOVA model, cTBS was assumed effective if group $\times$ time interaction was significant indicating more score changes in the experimental group compared to the control group. For all statistical analyses, a $\mathrm{P}<0.05$ was considered to be significant.

\section{Results}

Changes in the art constructive errors in painting

In our present study, the primary outcome was changes in the art constructive errors in painting (Table 2). All patients in both groups showed improvement in art constructive errors in painting effect (total error score of both the FCT and CT), revealed by repeated-measures ANOVA. In the repeated-measures ANOVA of art constructive errors in painting scores, the time factor was significant $(\mathrm{F}=130.567, \mathrm{P}=0.001)$ indicating that both groups' art constructive errors in painting scores (total error score) improved after the treatment. However, the group $\times$ time was not significant $(\mathrm{F}=1.612, \mathrm{P}=0.228)$ indicating no difference between $\mathrm{CTBS}+\mathrm{PA}$ and $\mathrm{PA}$ alone effect on art constructive errors in painting scores changes.

Figure 3 shows art constructive errors in painting scores' means in both groups before and after ten sessions of rehabilitation without painting practice. 


PA+CTBS group before

Figure 1. The classification of art constructive errors in the painting of patients with neglect used in this study

The figure on the left side of the columns shows the response of the patients with art constructive errors in painting in the Coloring test (CT) and neglect before the intervention. The patients changed often warm colors (yellow, light green, and red) to cold colors, which are characterized by cold, hard, and metallic colors, such as gray, black, dark green, brown, and marine blue (neglect in warm colors), and minimal color on the left side. Those on the right side of the columns are examples of the response of the patients with art constructive errors in painting in the $\mathrm{CT}$ and neglect of rehabilitation after 10 sessions. The patients used both warm colors and cold colors in the final paper

msc.b) Minimal size color on the left side errors before rehabilitation; msc. a) minimal size color on the left side errors after rehabilitation; cc.b) cold colors used (neglect of warm colors) before rehabilitation; cc.a) cold colors used (neglect of warm colors) after rehabilitation: oc.b: omission color errore before rehabilitation; oc.a, omission color errore after rehabilitation; pc.b)perseveration color errors before rehabilitation; Pc.a) perseveration color errors after rehabilitation; sc.b) small size copying errors before rehabilitation; sc.a) small size copying errors after rehabilitation; dc.b, deformation copying errors before rehabilitation; dc.a) deformation copying errors after rehabilitation; oco.b) omission copying errore before rehabilitation; oco.a) omission copying errore after rehabilitation; pco.b) perseveration copying errors before rehabilitation; pco.a) perseveration copying errors after rehabilitation; P.total errors b) total errors score of art constructive errors in painting before rehabilitation; P.total errors a) total errors score of art constructive errors in painting after rehabilitation; cTBS) continuous theta-burst transcranial magnetic stimulation.

Abbreviations PA: Prism adaptation; FCT: Figure copying test; CT: Coloring test; LBT: Line bisection test. 
All patients showed, on average, 5.3 (Mean \pm SD, 5.35 \pm 1.15 ) errors on art constructive errors of painting before rehabilitation compared to only 1.5 (Mean \pm SD $1.50 \pm 1.62$ ) errors on art constructive errors in painting after the intervention. Visuospatial omission, deformation errors, and neglect of warm colors (using cold colors) were the most frequent errors in the FCT, and CT followed by stroke patients with neglect in the pattern of painting' errors. A reduction was observed in the scores of all classifications of art constructive errors in painting, which suggests improvement in errors in painting symptoms in participants of both groups after the intervention (Figure 3 ).

Classification of art constructive errors in painting, omission, deformation, perseveration (addition), neglect of warm color (using cold color), and size errors were detected to be the sum of the scores of errors in the participant before and after the intervention (examples are shown in (Figures 1 and 2).

The results of increased significant improvement in the art constructive errors in painting and neglect are summarized in Figures 1.

\section{Changes in the neglect scores}

Neglect scores descriptive statistics before and after the rehabilitation are provided in Table 2 . In the repeatedmeasures ANOVA model with the LBT, FCT, and CT scores as the outcome variable, the time factor was significant (LBT: $\mathrm{F}=31.630, \mathrm{P}<0.001$ ), (FCT: $\mathrm{F}=64.438$, $\mathrm{P}=0.001)$, and $(\mathrm{CT}: \mathrm{F}=117.923, \mathrm{P}<0.001)$ indicating that both groups' neglect variable scores improved after the rehabilitation. However, the group $\times$ time effect was not significant in the $\mathrm{LBT}(\mathrm{F}=0.338, \mathrm{P}=0.572)$, FCT $(\mathrm{F}=2.959, \mathrm{P}=0.111)$, and $\mathrm{CT}(\mathrm{F}=4.493, \mathrm{P}=0.056)$ indicating no difference between the PA combined with cTBS group in the LBT, FCT, and CT scores changes compared to the PA group (Figure 3).

\section{Discussion}

This pilot study showed that in all stroke patients with neglect and art constructive errors in painting were improved after ten sessions of rehabilitation with PA alone and cTBS combined with PA. Art constructive errors in painting were classified into visuospatial omission error, deformation error, perseveration error, visuospatial small size errors, and neglect of warm colors (using cold colors) in stroke patients with neglect. The most frequent errors were visuospatial omission, deformation, and neglect of warm colors.
Prism adaption has been used for neglect treatment with variable success. However, our findings corroborate some previous trials reporting neglect approaches for rehabilitation (De Wit et al., 2018; Vaes et al., 2018). Improved visuospatial unilateral neglect symptoms as a result of rehabilitation may translate to improvements in the art constructive errors in painting. Some other clinical trials did not find such an effect for the prism (Barrett et al., 2012; Ten Brink et al., 2017; Turton et al., 2010). Differences in the clinical trials' design (prism glasses with six degrees of the visual field displacement compared to prism glasses with ten degrees in the current study), the employed evaluation (measured using "Aiming" compared to measured using "Where"), and the characteristics of the recruited stroke patients with visuospatial unilateral neglect can explain this disparity. Another explanation is the fact that all patients with neglect received a modification of prism adaption by mirror therapy at baseline. In contrast to prism adaption, mirror therapy showed improvement in visuospatial unilateral neglect with success (Pulyk \& Hyryavets, 2018). It was previously shown that mirror therapy three times a week for 20 minutes for three months performed better at the neglect test after the intervention compared to the control group. Mirror training acts by activating the mirror neuron system (Pulyk \& Hyryavets, 2018; Zhang et al., 2018)

Trials on cTBS rehabilitation approach over the left intact parietal cortex are effective therapies for neglect (Cazzoli et al., 2012; Cotoi et al., 2019). We found that a combination of PA and cTBS was effective in the art of constructive errors in painting and neglect recovery and was not even more efficacious when cTBS was added. One possible explanation is the near-complete rehabilitation effect of the art constructive errors in painting and neglect tests in both groups. All patients showed, on average, 5.3 errors in painting before rehabilitation compared to only 1.5 errors in painting after rehabilitation.

In the present study, it was hypothesized that the recovery combination stimulated and employed more networks of the brain, which might improve art constructive errors in painting and unilateral neglect in stroke patients. More studies are needed with functional and structural neuroimaging to verify this hypothesis. Previous studies have shown the promotion and correlation between changes in functional connectivity and structural attention network measured using neuroimaging in PA and cTBS (Fu et al., 2017; Nyffeler et al., 2019; Tsujimoto et al., 2019), and a reduction in pathological hyperexcitability (Corbetta \& Shulman, 2011; Koch et al., 2012; Koch et al., 2008), and the new mechanism of combined 


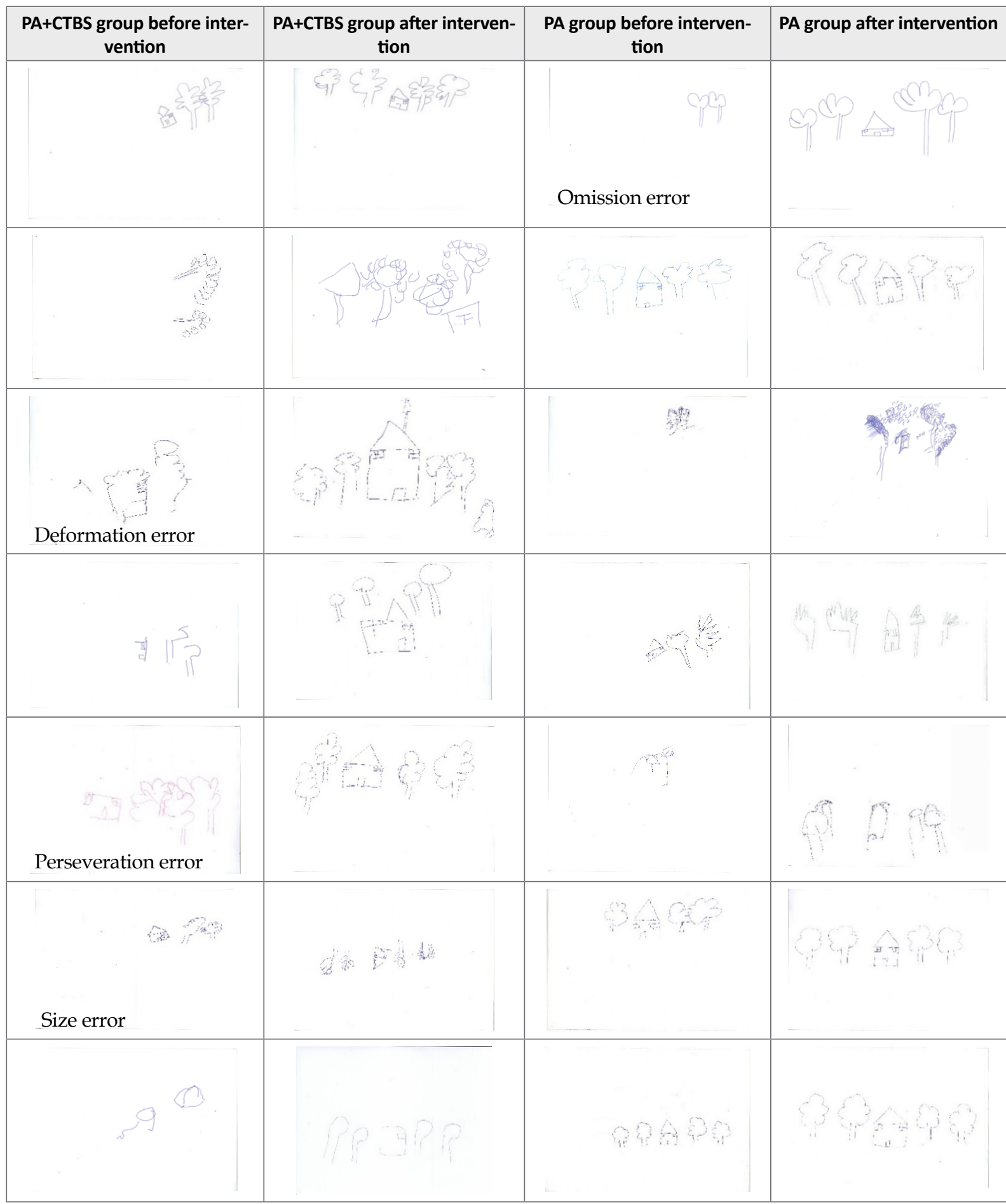

NEUR SCIENCE

Figure 2. The classification of art constructive errors in painting in the figure copying test (FCT) in stroke patients with neglect used in this study

The figure on the left side of the columns shows the response of the patients with art constructive errors in painting in FCT and neglect before the intervention. Those on the right side of the columns are examples of the response of the patients with art constructive errors in painting in FCT and neglect of rehabilitation after 10 sessions. 

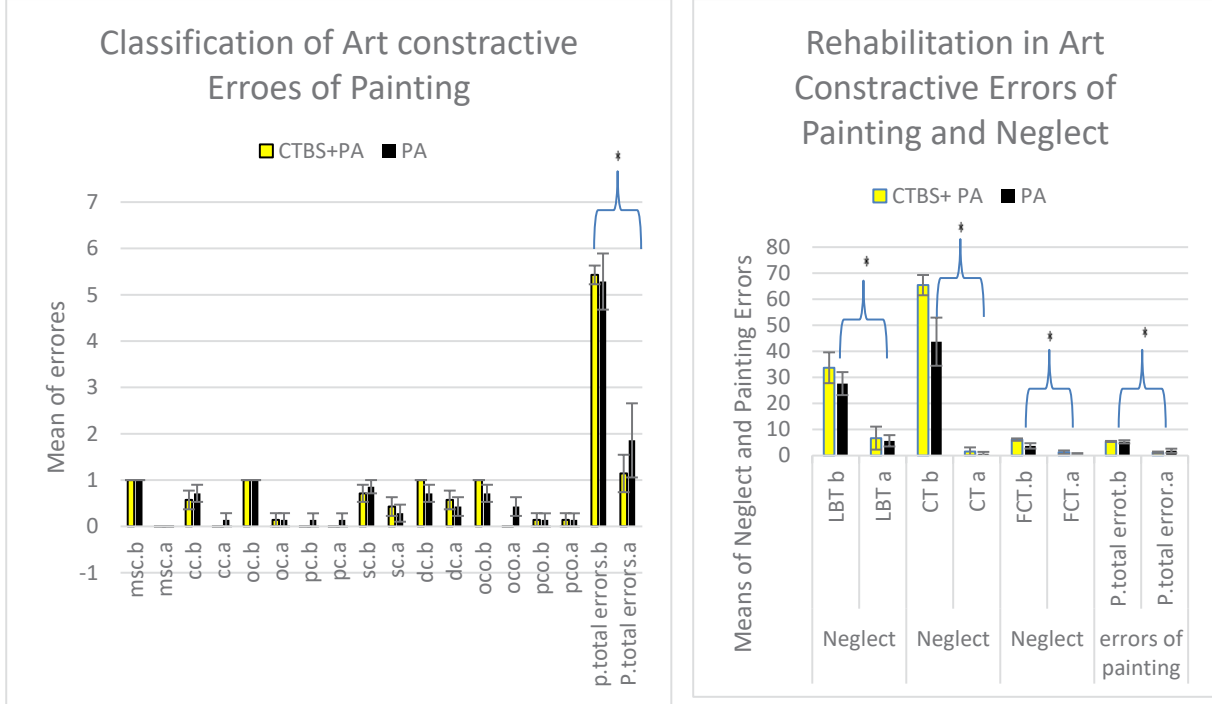

NEUR:SCIENCE

Figure 3. Art constructive errors in painting and visuospatial neglect measurement before and after the rehabilitation

The figure on the left side shows patients with the classification of art constructive errors of painting in the figure copying test and coloring test of rehabilitation without painting practice before and after 10 sessions. The figure on the right side shows stroke patients' scores in the neglect and art constructive errors in painting (total errors) of rehabilitation before and after 10 sessions. The rehabilitation significantly in art constructive errors in painting (measured using the pattern of painting' errors in FCT and CT), and neglect symptoms (measured by LBT, FCT, and CT). Asterisks indicate results that were significant using repeated-measures ANOVA ( ${ }^{*} \mathrm{P}<0.05$; time factor) and repeated-measures ANOVA (group $\times$ time factor; ${ }^{* *} \mathrm{P}<0.05$ ).

rehabilitation may explain the implicit recovery effect seen in stroke-induced neglect, and the art constructive errors in painting in the current study.

In contrast to implicit recovery (without practice) in the art constructive errors in painting, previous studies have been based on writing, imaging, painting, and relearning of practices (Pachalska et al., 2008). However, there is limited research into therapy for adults with acquired art constructive errors in painting. Previous studies on the art constructive errors in painting focused on stroke effects in professional painters (Pachalska et al., 2008; Rhee et al., 2016). Although patients in our study were not professional painters, they presented errors in the painting, like professional painters after a stroke.

Findings regarding the classification of constructive errors in painting showed that omission errors were most common in patients with stroke-induced neglect. Omission error outcome was consistent with studies on professional artists (Bäzner \& Hennerici, 2006; Bäzner \& Hennerici, 2007; Blanke \& Pasqualini, 2012; Rhee et al., 2016). Right-hemisphere stroke patients showed mostly left space omission errors and changes in painting style (Annoni et al., 2005; Bäzner \& Hennerici, 2007; Mazzucchi et al., 2013; Rhee et al., 2016). In contrast, left-hemisphere stroke patients showed painting without omission (Mazzucchi et al., 2013).

In our study, a wider use of cold colors (brown and marine blue) and minimal use of color on the left side was observed. Some patients with neglect used minimal color on the left whereas they colored the right side completely and evenly. Likewise, the selection of cold colors was observed before the intervention in professional artists with stroke-induced neglect (Bäzner \& Hennerici, 2006; Bäzner \& Hennerici, 2007; Blanke \& Pasqualini, 2012; Mazzucchi et al., 2013; Rhee et al., 2016). After rehabilitation, wider use of warm (yellow, light green, and red) and cold colors was observed in stroke patients with neglect.

In the current study, patients with neglect used minimal size in the drawing. However, our findings corroborate some previous studies reporting professional artists with neglect symptoms for a bi-dimensional perspective, in an attempt to avoid depth representation on their part of the painting (Bäzner \& Hennerici, 2006; Mazzucchi et al., 2013). Unreality painting outcome of small size, deformation, and neglect was consistent with studies in professional artists with right-hemisphere stroke (Annoni et al., 2005; Bäzner \& Hennerici, 2007; Bäzner \& Hennerici, 2007; Blanke \& Pasqualini, 2012; Mazzuc- 
chi et al., 2013; Rhee et al., 2016; Rode et al., 2017). In contrast, left-hemisphere stroke patients often remained hemiplegic (right-sided) and learned to hold the brush with their left hand with practice. The beginner-style painting persisted in patients (Mazzucchi et al., 2013). Disturbance in the estimation of distance-related deep neglect mechanisms may underlie the small size perception, omission, and deforming effect seen in art constructive errors in painting in stroke patients (Bäzner \& Hennerici, 2007; Rode et al., 2017). The brain processing after rehabilitation in visuospatial unilateral neglect may translate to improvements in the art of constructive errors in painting.

In the present study, patients with frontal damage had perseveration. Likewise, frontal-brain stroke in professional painters showed mostly perseveration (adding) errors (Bäzner \& Hennerici, 2006; Bäzner \& Hennerici, 2007; Blanke \& Pasqualini, 2012; Mazzucchi et al., 2013).

This pilot study had some limitations; only a single center was assessed; thus, the study had a relatively limited sample size. Also, $40 \%$ of our stroke patients were in the acute phase of recovery. Likewise, cTBS was reported useful in rehabilitation in terms of neglect in the acute phases in some clinical trials (Kim et al., 2013; Nyffeler et al., 2019). Nevertheless, there was no statistically significant difference between the two groups at baseline in the time since the onset of the acute phase (Table 1). The absence of cTBS alone for comparison was also a limitation in the present study; however, cTBS has been conducted in a previous study on neglect recovery (Cazzoli et al., 2012). More research is required to replicate our findings in a larger group.

The present study was the first clinical trial of a combination of PA and cTBS therapies for the art of constructive errors in painting without practice in painting and neglect symptoms. Further studies are suggested on the relationship between the art constructive errors in painting and underlying impaired perception mechanisms of the brain using electrophysiological and, functional imaging to verify whether brain regions are activated or deactivated.

\section{Conclusions}

An influence was found between art constructive errors in the painting system and neglect in stroke patients. Art constructive errors in painting patterns were determined to improve size discrepancy, omission, deformation, perseveration, and neglect of warm colors. The current results showed that art constructive errors in painting and neglect may be affected by rehabilitation and art educational strategies. Neglect appears to specifically affect the painting systems of stroke patients. The novel PA combined with cTBS and PA alone method could be potentially useful tools for rehabilitating patients with stroke-induced art constructive errors in painting and neglect.

\section{Ethical Considerations}

\section{Compliance with ethical guidelines}

The study was approved by the Ethics Committee of Iran University of Medical Sciences. All the subjects gave their informed consent before conducting the study [IR.IUMS.REC.1396.93012334]. This study was registered at the Iranian Registry of Clinical Trials (Code: IRCT20170423033606N3).

\section{Funding}

This work was supported by the Iran Cognitive Sciences and Technologies Council (Grant No.1446508).

\section{Authors' contributions}

Conceptualization and study design: Shole Vatanparasti; Statistical analysis, data analysis and interpretation: Anoshirvan Kazemnejad and Shole Vatanparasti; Drafting of the manuscript: Shole Vatanparasti; Critical revision of the manuscript for important intellectual content: Shole Vatanparasti; Supervision: Shahram Oveisgharan;

\section{Conflict of interest}

The authors declared no conflict of interest.

\section{Acknowledgments}

We would like to thank the National Brain Mapping Lab (NBML).

\section{References}

Annoni, J. M., Devuyst, G., Carota, A., Bruggimann, L., \& Bogousslavsky, J. (2005). Changes in artistic style after minor posterior stroke. Journal of Neurology, Neurosurgery $\mathcal{E}$ Psychiatry, 76(6), 797-803. [PMID] [PMCID] 
Annoni, J., Devuyst, G., Carota, A., Bruggimann, L., \& Bogousslavsky, J. (2005). Changes in artistic style after minor posterior stroke. Journal of Neurology, Neurosurgery \& Psychiatry, 76(6), 797-803. [DOI:10.1136/jnnp.2004.045492] [PMID] [PMCID]

Azouvi, P., Jacquin-Courtois, S., \& Luauté, J. (2017). Rehabilitation of unilateral neglect: Evidence-based medicine. Annals of Physical and Rehabilitation Medicine, 60(3), 191-197. [DOI:10.1016/j.rehab.2016.10.006] [PMID]

Barrett, A. M., Goedert, K. M., \& Basso, J. C. (2012). Prism adaptation for spatial neglect after stroke: Translational practice gaps. Nature Reviews Neurology, 8(10), 567-577. [DOI:10.1038/ nrneurol.2012.170] [PMID] [PMCID]

Bäzner, H., \& Hennerici, M. (2006). Stroke in painters. International Review of Neurobiology, 74, 165-191. [DOI:10.1016/S00747742(06)74013-2] [PMID]

Bäzner, H., \& Hennerici, M. G. (2007). Lovis corinth: Integrating hemineglect and spatial distortions. Frontiers of Neurology and Neuroscience, 22, 30-43. [DOI:10.1159/000102870] [PMID]

Bäzner, H., \& Hennerici, M. G. (2007). Painting after right-hemisphere stroke-case studies of professional artists. Frontiers of Neurology and Neuroscience, 22, 1-13. [DOI:10.1159/000102820] [PMID]

Blanke, O., \& Pasqualini, I. (2012). The riddle of style changes in the visual arts after interference with the right brain. Frontiers in Human Neuroscience, 5, 154. [DOI:10.3389/fnhum.2011.00154] [PMID] [PMCID]

Bonato, M., Priftis, K., Marenzi, R., \& Zorzi, M. (2008). Modulation of hemispatial neglect by directional and numerical cues in the line bisection task. Neuropsychologia, 46(2), 426-433. [DOI:10.1016/j.neuropsychologia.2007.08.019] [PMID]

Cazzoli, D., Müri, R. M., Schumacher, R., von Arx, S., Chaves, S., \& Gutbrod, K., et al. (2012). Theta burst stimulation reduces disability during the activities of daily living in spatial neglect. Brain, 135(Pt 11), 3426-3439. [DOI:10.1093/brain/ aws182] [PMID]

Chen, H., Pan, X., Lau, J. K. L., Bickerton, W. L., Pradeep, B., \& Taheri, M., et al. (2016). Lesion-symptom mapping of a complex figure copy task: A large-scale PCA study of the BCoS trial. NeuroImage. Clinical, 11, 622-634. [PMID] [PMCID]

Corbetta, M., \& Shulman, G. L. (2011). Spatial neglect and attention networks. Annual Review of Neuroscience, 34, 569-599. [DOI:10.1146/annurev-neuro-061010-113731] [PMID] [PMCID]

Cotoi, A., Mirkowski, M., Iruthayarajah, J., Anderson, R., \& Teasell, R. (2019). The effect of theta-burst stimulation on unilateral spatial neglect following stroke: A systematic review. Clinical Rehabilitation, 33(2), 183-194. [DOI:10.1177/0269215518804018] [PMID]

De Wit, L., Ten Brink, A. F., Visser-Meily, J. M. A., \& Nijboer, T. C. W. (2018). Does prism adaptation affect visual search in spatial neglect patients: A systematic review. Journal of Neuropsychology, 12(1), 53-77. [DOI:10.1111/jnp.12100] [PMID]

Fu, W., Cao, L., Zhang, Y., Huo, S., Du, J., \& Zhu, L., et al. (2017). Continuous theta-burst stimulation may improve visuospatial neglect via modulating the attention network: A rand- omized controlled study. Topics in Stroke Rehabilitation, 24(4), 236-241. [PMID]

Guariglia, P., Matano, A., \& Piccardi, L. (2014). Bisecting or not bisecting: This is the neglect question. Line bisection performance in the diagnosis of neglect in right brain-damaged patients. PloS One, 9(6), e99700. [PMID] [PMCID]

Hreha, K., Gillen, G., Noce, N., \& Nilsen, D. (2018). The feasibility and effectiveness of using prism adaptation to treat motor and spatial dysfunction in stroke survivors with multiple incidents of stroke. Topics in Stroke Rehabilitation, 25(4), 305-311. [PMID]

Johannsen, L., \& Karnath, H. O. (2004). How efficient is a simple copying task to diagnose spatial neglect in its chronic phase? Journal of Clinical and Experimental Neuropsychology, 26(2), 251256. [DOI:10.1076/jcen.26.2.251.28085] [PMID]

Kim, B. R., Chun, M. H., Kim, D. Y., \& Lee, S. J. (2013). Effect of high-and low-frequency repetitive transcranial magnetic stimulation on visuospatial neglect in patients with acute stroke: A double-blind, sham-controlled trial. Archives of Physical Medicine and Rehabilitation, 94(5), 803-807. [DOI:10.1016/j. apmr.2012.12.016] [PMID]

Koch, G., Bonnì, S., Giacobbe, V., Bucchi, G., Basile, B., \& Lupo, F., et al. (2012). Theta-burst stimulation of the left hemisphere accelerates recovery of hemispatial neglect. Neurology, 78(1), 24-30. [PMID]

Koch, G., Oliveri, M., Cheeran, B., Ruge, D., Lo Gerfo, E., \& Salerno, S., et al. (2008). Hyperexcitability of parietal-motor functional connections in the intact left-hemisphere of patients with neglect. Brain, 131(Pt 12), 3147-3155. [PMID] [PMCID]

Mazzucchi, A., Sinforiani, E., \& Boller, F. (2013). Focal cerebral lesions and painting abilities. Progress in Brain Research, 204, 71-98. [DOI:10.1016/B978-0-444-63287-6.00004-X] [PMID]

Ng, M. J., Singh, P., Pandian, J. D., Arora, R., \& Kaur, P. (2015) Mirror therapy in unilateral neglect after stroke (MUST trial): A randomized controlled trial. Neurology, 84(12), 1286. [DOI:10.1212/01.wnl.0000462978.57606.5a] [PMID]

Nyffeler, T., Vanbellingen, T., Kaufmann, B. C., Pflugshaupt, T., Bauer, D., \& Frey, J., et al. (2019). Theta burst stimulation in neglect after stroke: Functional outcome and response variability origins. Brain, 142(4), 992-1008. [DOI:10.1093/brain/ awz029] [PMID]

Pachalska, M., Grochmal-Bach, B., Wilk, M., \& Buliński, L. (2008). Rehabilitation of an artist after right-hemisphere stroke. Medical Science Monitor, 14(10), CS110-CS124. [PMID]

Blanke, O., \& Pasqualini, I. (2012). The riddle of style changes in the visual arts after interference with the right brain. Frontiers in Human Neuroscience, 5, 154. [PMID]

Pulyk, O. R., \& Hyryavets, M. V. (2018). Treatment for patients with neglekt after ischemic stroke. Wiadomosci lekarskie (Warsaw, Poland : 1960), 71(2 pt 1), 326-328. [PMID]

Rhee, E., Hong, C., Kim, Y. E., \& Lee, B. C. (2016). Changes in painting style by poststroke mania. Journal of Stroke, 18(1), 117-119. [PMID] [PMCID]

Rode, G., Pagliari, C., Huchon, L., Rossetti, Y., \& Pisella, L. (2017). Semiology of neglect: An update. Annals of Physical and Rehabilitation Medicine, 60(3), 177-185. [DOI:10.1016/j.rehab.2016.03.003] [PMID] 
Rossi, S., Ferro, M., Cincotta, M., Ulivelli, M., Bartalini, S., \& Miniussi, C., et al. (2007). A real electro-magnetic placebo (REMP) device for sham transcranial magnetic stimulation (TMS). Clinical Neurophysiology, 118(3), 709-716. [PMID]

Rossini, P. M., Burke, D., Chen, R., Cohen, L. G., Daskalakis, Z., \& Di Iorio, R., et al. (2015). Non-invasive electrical and magnetic stimulation of the brain, spinal cord, roots and peripheral nerves: Basic principles and procedures for routine clinical and research application. An updated report from an IFCN Committee. Clinical Neurophysiology, 126(6), 1071-1107. [PMID] [PMCID]

Ten Brink, A. F., Visser-Meily, J. M., \& Nijboer, T. C. (2015). Study protocol of 'Prism Adaptation in Rehabilitation': A randomized controlled trial in stroke patients with neglect. $B M C$ Neurology, 15, 5. [PMID]

Ten Brink, A. F., Visser-Meily, J. M. A., Schut, M. J., Kouwenhoven, M., Eijsackers, A. L. H., \& Nijboer, T. C. W (2017). Prism adaptation in rehabilitation? No additional effects of prism adaptation on neglect recovery in the subacute phase poststroke: A randomized controlled trial Neurorehabilitation and Neural Repair, 31(12), 1017-1028. [DOI:10.1177/1545968317744277] [PMID]

Tsujimoto, K., Mizuno, K., Nishida, D., Tahara, M., Yamada E., \& Shindo, S., et al. (2018). Correlation between changes in functional connectivity in the dorsal attention network and the after-effects induced by prism adaptation in healthy humans: A dataset of resting-state $\mathrm{fMRI}$ and pointing after prism adaptation. Data in Brief, 22, 583-589. [PMID] [PMCID]

Turton, A. J., O'Leary, K., Gabb, J., Woodward, R., \& Gilchrist, I. D. (2010). A single blinded randomised controlled pilot trial of prism adaptation for improving self-care in stroke patients with neglect. Neuropsychological Rehabilitation, 20(2), 180-196. [DOI:10.1080/09602010903040683] [PMID]

Vaes, N., Nys, G., Lafosse, C., Dereymaeker, L., Oostra, K., \& Hemelsoet, D., et al. (2018). Rehabilitation of visuospatial neglect by prism adaptation: Effects of a mild treatment regime. A randomised controlled trial. Neuropsychological Rehabilitation, 28(6), 899-918. [DOI:10.1080/09602011.2016.1208617] [PMID]

Vallar, G., Zilli, T., Gandola, M., \& Bottini, G. (2006). Productive and defective impairments in the neglect syndrome: Graphic perseveration, drawing productions and optic prism exposure. Cortex: A Journal Devoted to the Study of the Nervous System and Behavior, 42(6), 911-920. [PMID]

Yang, W., Liu, T. T., Song, X. B., Zhang, Y., Li, Z. H., \& Cui, Z. H., et al. (2015). Comparison of different stimulation parameters of repetitive transcranial magnetic stimulation for unilateral spatial neglect in stroke patients. Journal of the Neurological Sciences, 359(1-2), 219-225. [DOI:10.1016/j.jns.2015.08.1541] [PMID]

Zhang, J. J. Q., Fong, K. N. K., Welage, N., \& Liu, K. P. Y. (2018). The activation of the mirror neuron system during action observation and action execution with mirror visual feedback in stroke: A systematic review. Neural Plasticity, 2018, 2321045. [PMID] [PMCID] 